\title{
Coronavirus disease 2019 (COVID-19) in autoimmune and inflammatory conditions: clinical characteristics of poor outcomes
}

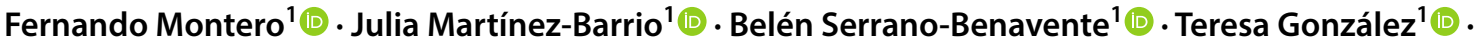

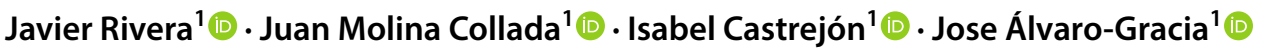

Received: 10 June 2020 / Accepted: 3 August 2020 / Published online: 13 August 2020

(c) Springer-Verlag GmbH Germany, part of Springer Nature 2020

\begin{abstract}
Objective To describe clinical characteristics of patients with rheumatic and musculoskeletal diseases (RMDs) and immunosuppressive therapies with Coronavirus disease 2019 (COVID-19) at an academic rheumatology center in Madrid and to identify baseline variables associated with a severe infection requiring hospitalization.

Methods We identified SARS-CoV-2 positive cases by polymerase chain reaction performed at our center within an updated RMDs database in our clinic. Additional RMDs patients were identified when they contacted the clinic because of a positive infection. Data extraction included diagnosis, demographics, immunosuppressive treatment, comorbidities, and laboratory tests. Comparisons between patients with or without hospitalization were performed. Multivariate logistic regression was used to analyze associations between baseline variables and need for hospitalization.

Results A total of 62 patients with COVID-19 and underlying RMDs were identified by April 24, 2020. Median age was 60.9 years, and $42 \%$ men. Forty-two patients required hospitalization; these were more frequently men, older and with comorbidities. There were no statistically significant between-group differences for rheumatologic diagnosis and for baseline use of immunosuppressive therapy except for glucocorticoids that were more frequent in hospitalized patients. Total deaths were $10(16 \%)$ patients. In multivariate analysis, male sex (odds ratio [OR], 8.63; $p=0.018)$, previous lung disease (OR, $27.47 ; p=0.042)$, and glucocorticoids use ( $>5 \mathrm{mg} /$ day) $(\mathrm{OR}, 9.95 ; p=0.019)$ were significantly associated to hospitalization. Conclusion Neither specific RMD diagnoses or exposures to DMARDs were associated with increased odds of hospitalization. Being male, previous lung disease and exposure to glucocorticoids were associated with higher odds of hospitalization in RMDs patients.
\end{abstract}

Keywords COVID-19 $\cdot$ Rheumatology $\cdot$ Autoimmune diseases $\cdot$ Poor outcomes

$\begin{array}{ll}\text { Abbreviations } \\ \text { COVID-19 } & \text { Coronavirus disease 2019 } \\ \text { RMDs } & \text { Rheumatic and musculoskeletal diseases. } \\ \text { PCR } & \text { Polymerase chain reaction } \\ \text { SLE } & \text { Systemic lupus erythematosus. } \\ \text { SS } & \text { Sjögren's syndrome. } \\ \text { SSc } & \text { Systemic sclerosis. } \\ \text { PMR } & \text { Polymyalgia rheumatica. } \\ \text { GCA } & \text { Giant cell arteritis. } \\ \text { SpA } & \text { Spondyloarthropathies. } \\ \text { CTD } & \text { Connective tissue diseases } \\ \text { OR } & \text { Odds ratio. }\end{array}$

Fernando Montero

fernandojose.montero@salud.madrid.org

1 Department of Rheumatology. Hospital General, Universitario Gregorio Marañón, 28007 Madrid, Spain
CI Confidence interval.

RA Rheumatoid arthritis.

DMARDs Disease-modifying antirheumatic drugs.

IQR Interquartile range

SD Standard deviation

\section{Introduction}

On December 31, 2019, the first cases of pneumonia of a new type of virus in the family Coronaviridae, named SARS-CoV-2, were reported in Wuhan. So far, more than 10.000.000 cases have been detected worldwide [1]. The first patient infected with SARS-CoV-2 virus in Spain was identified in January 2020, being Madrid the city with the greatest impact, with 72.000 confirmed cases and more than 8300 deceased by May 2020 [2]. 
Individuals with rheumatic and musculoskeletal diseases (RMDs) and immunosuppressive therapies may require special consideration because receiving immunosuppressant therapies made them more susceptible to viral and bacterial infections. However, to date, it has not shown a higher incidence of Coronavirus disease 2019 (COVID-19) in patients with rheumatic diseases compared to the general population [3], even if receiving biologic disease-modifying antirheumatic drug (b-DMARD) therapy $[4,5]$. In addition, a preliminary survey study showed that patients treated with DMARDs and infected with COVID-19 did not develop lifethreatening complications due to their underlying medication [6].

Multiple guidelines from different scientific societies have been published with recommendations for COVID-19 in rheumatic patients. It seems that the risk of severe outcomes in patients with rheumatic diseases and COVID-19, is tied to age and comorbidities as in the general population $[7,8]$. However, as comorbidities occur more frequently in patients with rheumatic disease as a result of the baseline disease or as a complication of treatment, there is a major need to determine which therapies or baseline factors may increase the vulnerability to infection and may predict poorer outcomes.

Treatments commonly used in patients with rheumatic disease have been shown useful to treat COVID-19. For example, as interleukin (IL)-6 is a cytokine highly expressed in severe forms of COVID-19 associated to the cytokine storm [9], IL-6 inhibitors are currently being used in the treatment of severe COVID-19. It is not clear if IL-6 inhibitors already prescribed in rheumatic patients may have a protective effect.

The aim of our study was to describe epidemiological and clinical characteristics of patients with rheumatic and musculoskeletal diseases (RMDs), treated with immunosuppressive therapies presenting COVID-19 in a reference hospital in Spain, and to explore baseline variables, included diagnosis and baseline therapy, associated with a more severe infection requiring hospitalization.

\section{Patients and methods}

Patients We conducted a retrospective observational study including patients with any rheumatologic autoimmune or inflammatory diseases evaluated in our rheumatology department and who were infected with SARS-CoV2 between March 4 and April 24, 2020.

In our department, we maintain an updated database of patients with any rheumatologic autoimmune disease, including systemic lupus erythematosus (SLE), Sjögren's syndrome (SS), systemic sclerosis (SSc), polymyalgia rheumatica (PMR), giant cell arteritis (GCA) and other systemic vasculitis and inflammatory myopathies. We identified SARS-CoV-2 positive by polymerase chain reaction (PCR) performed at our center with our database of rheumatology patients evaluated at our clinic and with an updated registry of patients on active biological therapy from January 2020, mostly patients with rheumatoid arthritis (RA) and spondyloarthritis $(\mathrm{SpA})$ who were receiving any of the following treatments: anti-TNF alpha drugs (etanercept, adalimumab, infliximab, golimumab and certolizumab), IL-1 inhibitors (anakinra), IL-6 inhibitors (tocilizumab), IL 12/23 inhibitors (ustekinumab), IL-17 inhibitors (secukinumab), CTLA4-Ig (abatacept), JAK inhibitors (tofacitinib, baricitinib and ruxolitinib). Additional RMDs patients were identified when they contacted our clinic to inform us about a positive COVID-19 infection.

A diagnosis of SARS-CoV2 was considered if the patient had a positive polymerase chain reaction (PCR) test on respiratory samples during the study time frame or if there were high suspicions of infection [Compatible respiratory symptoms (dyspnea, cough, anosmia, ageusia, low $\mathrm{O}_{2}$ saturations) with radiographic images of pneumonic infiltrates with hospitalization and/or therapy initiation specific for COVID-19].

We collected the following data from the electronic health records: demographic characteristics, comorbidities, and clinical characteristics of the infection, laboratory test data, and outcome (dead, hospital admission). The "standard treatment" for COVID-19 offered at our institution included hydroxychloroquine and/or lopinavir-ritonavir and/or azithromycin, and for patients requiring hospitalization oxygen therapy, glucocorticoids, remdesivir, anti-interleukine-6 (tocilizumab), and anti-interleukine-1 (anakinra) may be also added for treatment intensification.

Baseline treatment-related data were extracted from the electronic health records for type of DMARD prescribed (i.e., methotrexate, hydroxychloroquine, leflunomide, sulfasalazine), biologic treatment, and prednisone use and dose in milligrams. Time from symptom onset to PCR testing was also calculated. Any radiographic evidence of pneumonia at the medical center was also recorded.

Statistical analysis The results of descriptive analyses were presented using frequencies and percentages for qualitative variables and as median or mean values for continuous variables. The patients were classified in two groups according to the severity of COVID-19 infection requiring or not requiring hospitalization. Between-group comparisons were made using Student's $t$ test to analyze differences between means; chi-square tests were used for comparisons between proportions. Nonparametric tests (i.e., Kruskal-Wallis) were used for data that did not meet the assumptions of a normal distribution. Normality in distribution was tested by graphical and numerical methods. A histogram plot and Skewness/ Kurtosis tests were computed for each variable. 
We used a bivariate logistic regression model to assess factors associated with a more severe infection requiring hospitalization. Age higher than 70, being male, having RA, SpA or SLE in comparison with other rheumatic diagnosis, comorbidities, and baseline therapy were evaluated for possible association with a more severe infection requiring hospitalization. Any factor with a $p$ value $<0.15$ in the bivariate analysis was included in the multivariate analysis. The multivariable logistic regression model first included all variables that were significant; backward elimination was then used to select the final model.

All statistical tests were two-sided; $p$ values $<0.05$ were considered to indicate a statistically significant result. STATA $12.0 ®$ for Mac (StataCorp LP, College Station, TX) software was used for all analyses.

\section{Ethics and institutional review board approval}

This study was performed in accordance with the ethical standards of the responsible committee on human experimentation and the Helsinki Declaration of 1975, as revised in 2013. All data were anonymised, and the study was approved by the Ethics Committee of Hospital General Universitario Gregorio Marañón (April 27th, ref $\mathrm{N}^{\circ} \mathrm{CEIm}$ : 12/2020).

\section{Results}

We identified 62 patients with COVID-19 between March 4 and April 24, 2020 with a follow-up in our department for a rheumatologic autoimmune or inflammatory disease.

Forty-two patients (68\%) were hospitalized and $20(32 \%)$ were followed up at in-home isolation, out of the total of 62 patients, 51 had a positive PCR. Patient characteristics are presented in Table 1. The patient's mean age was 60.9 years, $42 \%$ were men. The most common rheumatologic diagnosis was RA (32\%) followed by SpA (26\%). The most common comorbidities were cardiovascular disease (52\%), and hypertension $(45 \%)$.

Male gender, cardiovascular disease, hypertension, and diabetes were associated with a more severe infection requiring hospital admission $(p \leq 0.05)$. No statistically significant differences were observed between the two groups (hospitalized versus non-hospitalized) with regard to rheumatologic diagnosis and use of immunosuppressive drugs except for glucocorticoids that were more frequently used in patients requiring hospitalization. Around one-third of patients not requiring hospitalization were health care personnel. Eightyseven percent $(87 \%)$ of hospitalized patients have radiograph findings compatible with pneumonia and received a more intensive treatment in line with higher severity. Azithromycin, hydroxychloroquine, lopinavir/ritonavir and tocilizumab were prescribed either in monotherapy or in combination and tocilizumab was used in $5(12 \%)$ hospitalized patients. No statistical differences were observed between the two groups in terms of mortality; however, death clearly tended to be more frequent in the hospitalized group (21\% versus $5 \%, p=0.14)$.

A total of 10 patients died (16\%), most of them (9 patients) in the hospitalized group: 3 with RA, 2 with LES, 1 with SpA and 4 with other rheumatic diseases. Patients who died were older (72.8 years old versus $59.9, p<0.001$ ), most of them were men $(70 \%)$, and had more comorbidities, mainly DM (60\%) and HTA (80\%).

Results from the multivariate analyses (Table 2) revealed an association between male sex, preexisting lung disease and treatment with glucocorticoids ( $\geq 5 \mathrm{mg}$ per day) with a more severe infection requiring hospitalization.

\section{Discussion}

In the present study, we report how patients with inflammatory and autoimmune rheumatic diseases from our center were affected by COVID-19 infection. Our results support that male sex, preexisting lung disease and baseline treatment with glucocorticoids ( $>5 \mathrm{mg} /$ day) are associated with higher odds of a more severe infection requiring COVID-19 hospitalization. We did not find association between hospitalization and specific rheumatic diagnosis or baseline therapy. The rate of hospitalization was high in our cohort (68\%), reflecting identification by PCR positivity and probably underestimating less severe cases in patients who did not reach medical care.

Our results are in line with previously described in the literature, alerting the importance of comorbidities like hypertension or pulmonary disease regarding COVID19 infection severity in the general population $[8,10-12]$ but also in series of patients with rheumatic diseases [7]. We did not find any association between baseline immunosuppressive drugs and hospitalization except for use of glucocorticoids. According to data from 600 patients in the COVID-19 Global Rheumatology Alliance registry showed similar results [7], most immunosuppressive drugs, including biologics and targeted synthetic agents, were not associated with poorer outcomes. Similar results have been found in a case series of 86 patients with COVID-19 in New York [13], and in two age and sex-matched control cohorts [3, 14]. Similarly, Monti et al. [15], and Conticini et al. [6] described low incidence of SARS-CoV2 infection in patients with immunosuppressive drugs, suggesting low influence of these medications in acquiring the illness and course severity.

About glucocorticoids influence on COVID-19 evolution, although they could be an alternative therapeutic strategy when distress respiratory syndrome overcome, a 
Table 1 Comparison of demographic, baseline clinical characteristics and outcomes of patients with different rheumatic conditions infected with COVID-19 by hospitalization

\begin{tabular}{|c|c|c|c|c|}
\hline & $\begin{array}{l}\text { Total } \\
N=62\end{array}$ & $\begin{array}{l}\text { Non-hospitalized } \\
N=20(32 \%)\end{array}$ & $\begin{array}{l}\text { Hospitalized } \\
N=42(68 \%)\end{array}$ & $p$ \\
\hline Age, mean (SD) & $60.9(13.9)$ & $56.4(10.8)$ & $63.0(14.9)$ & 0.08 \\
\hline Patients $\geq 70$ years, number $(\%)$ & $18(29 \%)$ & $2(10 \%)$ & $16(38 \%)$ & 0.02 \\
\hline Male, number (\%) & $26(42 \%)$ & $4(20 \%)$ & $22(52 \%)$ & 0.01 \\
\hline \multicolumn{5}{|l|}{ Rheumatic diagnosis } \\
\hline Rheumatoid Arthritis & $20(32 \%)$ & $5(25 \%)$ & $15(36 \%)$ & \multirow[t]{5}{*}{0.60} \\
\hline $\mathrm{SpA} /$ psoriatic arthritis & $16(26 \%)$ & $5(25 \%)$ & $11(26 \%)$ & \\
\hline Other inflammatory & $4(6 \%)$ & $1(5 \%)$ & $3(7 \%)$ & \\
\hline SLE & $9(15 \%)$ & $3(15 \%)$ & $6(14 \%)$ & \\
\hline Other CTD & $13(21 \%)$ & $6(30 \%)$ & $7(17 \%)$ & \\
\hline Disease duration, years, mean (SD) & $11.1(8.5)$ & $10.6(8.7)$ & $11.3(8.5)$ & 0.74 \\
\hline \multicolumn{5}{|l|}{ Comorbidities } \\
\hline Obesity & $20(33 \%)$ & $4(20 \%)$ & $16(40 \%)$ & 0.12 \\
\hline Diabetes mellitus & $12(20 \%)$ & $0(0 \%)$ & $12(30 \%)$ & 0.006 \\
\hline Hypertension & $27(45 \%)$ & $4(20 \%)$ & $23(57 \%)$ & 0.006 \\
\hline Cardiovascular disease & $31(52 \%)$ & $6(30 \%)$ & $25(62 \%)$ & 0.01 \\
\hline Lung disease & $14(23 \%)$ & $1(5 \%)$ & $13(32 \%)$ & 0.01 \\
\hline \multicolumn{5}{|l|}{ Baseline therapy } \\
\hline Glucocorticoids, $n(\%)$ & $30(48 \%)$ & $5(25 \%)$ & $25(59 \%)$ & 0.01 \\
\hline Dose $\geq 5 \mathrm{mg}, n(\%)$ & $27(44 \%)$ & $4(20 \%)$ & $23(55 \%)$ & 0.01 \\
\hline \multicolumn{5}{|l|}{ Non-biologics DMARDS } \\
\hline Hydroxychloroquine & $9(14 \%)$ & $3(15 \%)$ & $6(14 \%)$ & 0.94 \\
\hline Methotrexate & $12(19 \%)$ & $2(10 \%)$ & $10(24 \%)$ & 0.19 \\
\hline Leflunomide & $3(5 \%)$ & $0(0 \%)$ & $3(7 \%)$ & 0.22 \\
\hline \multicolumn{5}{|l|}{ Biologics } \\
\hline Anti-TNF & $12(19 \%)$ & $5(25 \%)$ & $7(17 \%)$ & 0.43 \\
\hline Tocilizumab & $4(6 \%)$ & $2(10 \%)$ & $2(5 \%)$ & 0.43 \\
\hline Tofacitinib & $2(3 \%)$ & $1(5 \%)$ & $1(2 \%)$ & 0.58 \\
\hline Positive PCR for SARS-CoV2 (respiratory samples) & $51(82 \%)$ & $15(75 \%)$ & $36(86 \%)$ & 0.30 \\
\hline Health Care Personnel & $7(13 \%)$ & $6(33 \%)$ & $1(3 \%)$ & 0.002 \\
\hline \multicolumn{5}{|l|}{ Clinical features } \\
\hline C-reactive protein $(\mathrm{mg} / \mathrm{dL})$, median (IQR) & $3.6(1.7-10.8)$ & $1.5(1.4-2.1)$ & $4.4(1.8-10.9)$ & 0.09 \\
\hline Lymphocytes $\left(/ \mathrm{mm}^{3}\right)$, median $(\mathrm{IQR})$ & $1100(700-1700)$ & $1850(1400-1900)$ & $1050(700-1500)$ & 0.05 \\
\hline Pneumonia by chest X-ray & $36(64 \%)$ & $1(6 \%)$ & $35(87 \%)$ & $<0.001$ \\
\hline \multicolumn{5}{|l|}{ COVID-19 therapy, number (\%) } \\
\hline Hydroxychloroquine & $40(64 \%)$ & $3(15 \%)$ & $37(88 \%)$ & 0.001 \\
\hline Lopinavir/ritonavir & $36(58 \%)$ & $3(15 \%)$ & $33(79 \%)$ & 0.001 \\
\hline \multicolumn{5}{|l|}{ Glucocorticoids } \\
\hline Azithromycin & $9(15 \%)$ & $0(0 \%)$ & $9(21 \%)$ & 0.02 \\
\hline Tocilizumab & $5(8 \%)$ & $0(0 \%)$ & $5(12 \%)$ & 0.10 \\
\hline \multicolumn{5}{|l|}{ Outcome, number (\%) } \\
\hline Death & $10(16 \%)$ & $1(5 \%)$ & $9(21 \%)$ & 0.14 \\
\hline
\end{tabular}

$C T D$ connective tissue diseases, $D M A R D$ disease-modifying antirheumatic drugs, $I Q R$ Interquartile range, $P C R$ polymerase chain reaction, $S L E$ systemic lupus erythematosus, $S p A$ spondyloarthropathies, $S D$ standard deviation, $T N F$ tumor necrosis factor

possible role in decreasing viral clearance in the initial stage of the disease has been suggested $[11,16,17]$ and may be related to our findings. Uses of glucocorticoids have been associated with a higher risk of hospitalization and severe outcomes also in patients with inflammatory bowel diseases [18]. Our data are in agreement with other studies and suggest that glucocorticoids early in infection 
Table 2 Associations between baseline characteristics and more severe infection requiring hospitalization; results of bivariate and multivariate logistic regression analyses $(n$ $=62$ )

\begin{tabular}{|c|c|c|c|c|}
\hline \multirow[t]{2}{*}{ Variables } & \multicolumn{2}{|l|}{ Bivariate analyses } & \multicolumn{2}{|c|}{ Multivariate analyses } \\
\hline & OR $(95 \% \mathrm{CI})$ & $P$ values & $\overline{\mathrm{OR}}(95 \% \mathrm{CI})$ & $P$ values \\
\hline Age $\geq 70$ years & $5.50(1.13-27.10)$ & 0.035 & $2.6(0.42-16.21)$ & 0.303 \\
\hline Men $($ Women $=$ reference $)$ & $4.40(1.25-15.39)$ & 0.020 & $7.4(1.58-34.90)$ & 0.011 \\
\hline RA & $1.67(0.50-5.49)$ & 0.401 & - & - \\
\hline SPA & $1.06(0.31-3.62)$ & 0.920 & - & - \\
\hline SLE & $0.94(0.21-4.24)$ & 0.941 & - & - \\
\hline Obesity & $2.66(0.75-9.44)$ & 0.129 & $0.64(0.09-4.48)$ & 0.656 \\
\hline Hypertension & $5.41(1.53-19.12)$ & 0.009 & $6.21(0.58-66.62)$ & 0.579 \\
\hline Cardiovascular disease & $3.89(1.23-12.29)$ & 0.021 & $0.73(0.12-4.54)$ & 0.738 \\
\hline Lung disease & $9.14(1.10-75.98)$ & 0.040 & $8.93(1.25-63.48)$ & 0.029 \\
\hline Glucocorticoids dose $\geq 5 \mathrm{mg} /$ day & $4.84(1.38-16.95)$ & 0.014 & $5.00(1.08-23.15)$ & 0.040 \\
\hline
\end{tabular}

$C I$ confidence interval, $O R$ odds ratio, $R A$ rheumatoid arthritis, $S L E$ systemic lupus erythematosus, $S p A$ spondyloarthropathies are harmful even though some studies suggest a significant benefit later during the COVID-19 course [19].

Interestingly, reported data from the COVID-19 Global Rheumatology Alliance registry [7] showed a lower mortality among rheumatologic patients when comparing with our series. Possible explanations are the lower rate of older patients with only $18 \%$ older than 65 in the former series and the lower proportion of hospitalized patients (35\% vs 68\%). In addition, Madrid was one of the most affected regions by the COVID-19 pandemic in Spain with a considerable rate of severe cases.

Our study has several limitations, as it is a retrospective single-center study, with a small sample and short period study that could limit more conclusive results. Also, a potential selection bias for more severe cases is to be considered. Nevertheless, it is an initial approach to know how COVID-19 infection behave in rheumatic patients and the risk factors associated to a worse prognosis, made in a homogeneous patient group.

In conclusion, age and previous lung disease must be carefully assessed when treating COVID-19 patients with inflammatory and autoimmune rheumatic diseases. More research is needed to evaluate specific immunosuppressive medication and other comorbidity interactions; but according to our data and in agreement with other studies, patients with rheumatic disease do not need to discontinue immunosuppressive drugs. Because of the association of glucocorticoids with poorer outcomes, it is important to minimize exposure.

Results from other studies are expected to further understand the impact of COVID-19 in rheumatologic patients, specially concerning long-term outcomes.

Acknowledgements We are grateful to all rheumatologists at HGU Gregorio Marañón who help identifying patients with COVID-19 in their clinics and the Microbiology Department for providing registers of PCR making this study possible.

Author contributions JMB, IC and JMAG have made substantial contributions to the conception and design of the work; FM, BSB, and IC have participated in the acquisition and analysis of the data. All authors have contributed to the interpretation of data for the work and revising it critically for important intellectual content and final approval.

Funding This research was supported by internal funding and not by any specific grants from funding agencies in the public, commercial, or not-for-profit sectors.

Availability of data and materials The datasets used and/or analyzed during the current study are available from the corresponding author on reasonable request.

\section{Compliance with ethical standard}

Conflict of interest The authors declare that they have no competing interest. Patient and public involvement Patients and/or the public were not involved in the design, or conduct, or reporting, or dissemination plans of this research. Patient consent for publication: waived by the local IRB.

\section{References}

1. Zhu N, Zhang D, Wang W et al (2019) (2020) A Novel Coronavirus from Patients with Pneumonia in China. N Engl J Med 382(8):727-733

2. Gobierno de España. Ministerio de Sanidad. Secretaria General de sanidad. Dirección General de salud pública, calidad e información. Centro de Coordinación de Alertas y Emergencias Sanitarias. (Government of Spain. Ministry of Health. General Secretary of Health. General Directorate of Public Health, Quality and Information. Center for Coordination of Health Alerts and Emergencies) (2020). Enfermedad por el coronavirus (COVID-19). (Coronavirus disease (COVID-19)) Actualización no 104. https:// 
www.mscbs.gob.es/profesionales/saludPublica/ccayes/alertasAct ual/nCov-China/documentos/Actualizacion_104_COVID-19.pdf

3. Pablos JL, Abasolo L, Alvaro-Gracia JM et al (2020) Prevalence of hospital PCR-confirmed COVID-19 cases in patients with chronic inflammatory and autoimmune rheumatic diseases. Ann Rheum Dis. https://doi.org/10.1136/annrheumdis-2020-217763

4. Michelena X, Borrell H, Lopez-Corbeto M et al (2020) Incidence of COVID-19 in a cohort of adult and paediatric patients with rheumatic diseases treated with targeted biologic and synthetic disease-modifying anti-rheumatic drugs. Semin Arthritis Rheum. https://doi.org/10.1016/j.semarthrit.2020.05.001

5. Figueroa-Parra G, Aguirre-Garcia GM, Gamboa-Alonso CM, Camacho-Ortiz A, Galarza-Delgado DA (2020) Are my patients with rheumatic diseases at higher risk of COVID-19? Ann Rheum Dis. https://doi.org/10.1136/annrheumdis-2020-217322

6. Conticini E, Bargagli E, Bardelli M et al (2020) COVID-19 pneumonia in a large cohort of patients treated with biological and targeted synthetic antirheumatic drugs. Ann Rheum Dis. https:// doi.org/10.1136/annrheumdis-2020-217681

7. Gianfrancesco MA, Hyrich KL, Gossec L et al (2020) Rheumatic disease and COVID-19: initial data from the COVID-19 Global Rheumatology Alliance provider registries. Lancet Rheumatol. https://doi.org/10.1016/S2665-9913(20)30095-3

8. Ruan Q, Yang K, Wang W, Jiang L, Song J (2020) Clinical predictors of mortality due to COVID-19 based on an analysis of data of 150 patients from Wuhan China. Intensive Care Med 46(5):846-848

9. Ye Q, Wang B, Mao J (2020) The pathogenesis and treatment of the 'Cytokine Storm' in COVID-19. J Infect 80(6):607-613

10. Zhou F, Yu T, Du R et al (2020) Clinical course and risk factors for mortality of adult inpatients with COVID-19 in Wuhan, China: a retrospective cohort study. Lancet 395(10229):1054-1062

11. Wu C, Chen X, Cai Y et al (2020) Risk factors Associated with acute respiratory distress syndrome and death in patients with coronavirus disease 2019 pneumonia in Wuhan China. JAMA Intern Med. https://doi.org/10.1001/jamainternmed.2020.0994
12. Chen N, Zhou M, Dong X et al (2020) Epidemiological and clinical characteristics of 99 cases of 2019 novel coronavirus pneumonia in Wuhan, China: a descriptive study. Lancet 395(10223):507-513

13. Haberman R, Axelrad J, Chen A et al (2020) Covid-19 in ImmuneMediated Inflammatory Diseases - Case Series from New York. N Engl J Med 383(1):85-88

14. D'Silva KM, Serling-Boyd N, Wallwork R et al (2020) Clinical characteristics and outcomes of patients with coronavirus disease 2019 (COVID-19) and rheumatic disease: a comparative cohort study from a US 'hot spot'. Ann Rheum Dis. https://doi. org/10.1136/annrheumdis-2020-217888

15. Monti S, Balduzzi S, Delvino P, Bellis E, Quadrelli VS, Montecucco C (2020) Clinical course of COVID-19 in a series of patients with chronic arthritis treated with immunosuppressive targeted therapies. Ann Rheum Dis 79(5):667-668

16. Stockman LJ, Bellamy R, Garner P (2006) SARS: systematic review of treatment effects. PLoS Med 3(9):e343

17. Russell CD, Millar JE, Baillie JK (2020) Clinical evidence does not support corticosteroid treatment for 2019-nCoV lung injury. Lancet 395(10223):473-475

18. Brenner EJ, Ungaro RC, Gearry RB et al (2020) Corticosteroids, but not TNF antagonists, are associated with adverse COVID-19 outcomes in patients with inflammatory bowel diseases: results from an international registry. Gastroenterology. https://doi. org/10.1053/j.gastro.2020.05.032

19. Ledford $H$ (2020) Coronavirus breakthrough: dexamethasone is first drug shown to save lives. Nature 582(7813):469

Publisher's Note Springer Nature remains neutral with regard to jurisdictional claims in published maps and institutional affiliations. 